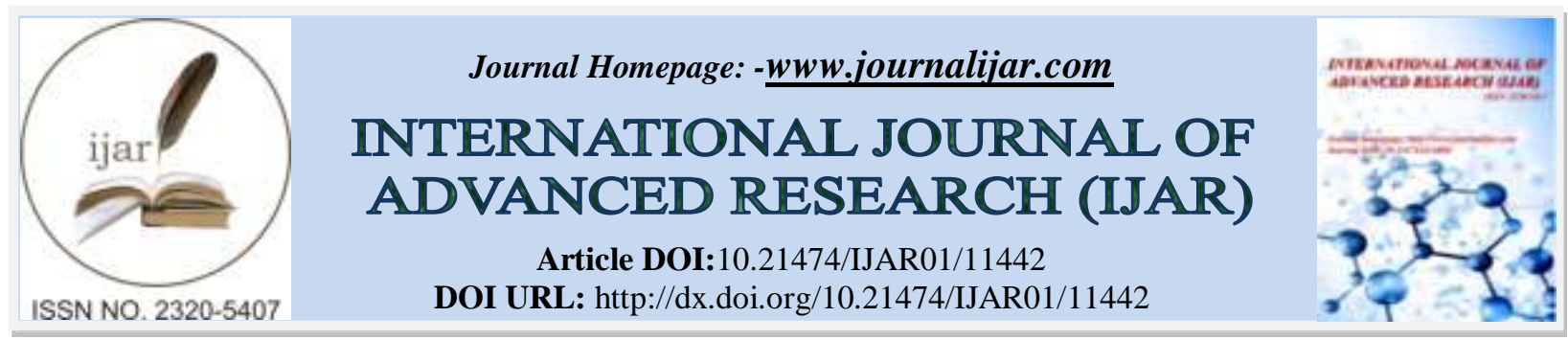

RESEARCH ARTICLE

\title{
A CLINICOMYCOLOGICAL STUDY OF DERMATOPHYTE INFECTION IN A TERTIARY CARE HOSPITAL AT GWALIOR - A PROSPECTIVE ANALYSIS
}

\author{
Priyanka Gupta $^{1}$, Vaibhav Misra ${ }^{2}$, Savita Bharat Jain ${ }^{3}$ and Rishu Kumari ${ }^{4}$ \\ 1. Post Graduate student, Department of Microbiology, Gajra Raja Medical College, Gwalior, Madhya Pradesh. \\ 2. Professor, Department of Microbiology, Gajra Raja Medical College, Gwalior, Madhya Pradesh. \\ 3. Professor and Head, Department of Microbiology, Gajra Raja Medical College, Gwalior, Madhya Pradesh. \\ 4. MD, Microbiology.
}

\section{Manuscript Info}

Manuscript History

Received: 31 May 2020

Final Accepted: 30 June 2020

Published: July 2020

Key words:-

Dermatophytosis, Tinea Corporis,

Trichophyton Rubrum

\section{Abstract}

Introduction : Dermatophytosis are the most common type of superficial fungal infection seen in humans caused by keratinophilic fungi, capable of invading keratinized tissues of skin and its appendages.Tinea corporis is the commonest clinical type of dermatophyte in India followed by Tinea cruris. The distribution and frequency of Dermatophytosis and their etiologic agents vary according to the geographic region studied, the socioeconomic level of the population, the climatic variations, the presence of domestic animal and age of the individual.

Objectives: This study was undertaken to isolate and identify the fungal pathogen causing dermatophytosis in patients attending skin OPD at J.A Group of Hospitals, Gwalior (M.P).

Material and Methods : We prospectively analyzed 300 samples of dermatophytosis in our tertiary care hospital.Skin scrapings, hair and nails were collected and processed and fungal pathogens were identified by standard laboratory techniques.

Result : Out of 300 Samples, 201 (67\%) were culture positive among which prevalence of dermatophyte was maximum i.e 159 (53\%) while (42)14\% were non dermatophytic fungus and candida spp. Tinea corporis was the predominant dermatomycotic lesion accounted for 143 $(47.67 \%)$ cases followed by tinea cruris $46(15.33 \%)$, Tinea manuum $17(5.67 \%)$, Tinea pedis $11(3.66 \%)$, Tinea faciei was seen in $3(1 \%)$ cases. $29(9.67 \%)$ were from Tinea capitis, $6(2 \%)$ were from Tinea barbae, while Tinea unguium was $45(15 \%)$.

Conclusion: Tinea corporis was the predominant clinical site from which dermatophytes were isolated.Trichophyton rubrum was the predominant species isolated from Tinea corporis, Tinea cruris, Tinea unguium and Tinea capitis.

Copy Right, IJAR, 2020,. All rights reserved.

\section{Introduction:-}

Dermatophytosis are the most common type of superficial fungal infection seen in humans caused by group of closely related keratinophilic fungi, capable of invading keratinized tissues of skin and its appendages. ${ }^{1}$ by 
producing proteases enzyme that digest and use keratin as a source of nitrogen and permits infection, colonization and invasion of the stratum corneum of the skin and its appendages. ${ }^{2}$ Infection is restricted to the dead cornified layers only. ${ }^{3-4}$ Trichophyton, Microsporum and Epidermophyton, are the three major genera of the class Hyphomycetes and division Deuteromycota. ${ }^{5}$ The typical infections of Dermatophytes are referred to as 'ringworm infections' due to their ring like appearance and also known as 'Tinea infections'.The prevalence of Dermatophytosis varies in India, more prevalence in southern and eastern region than the northern regions of the country. Tinea cruris and Tinea corporis are the commonest varieties seen in India followed by Tinea capitis, Tinea pedis, Tinea barbae, Tinea unguium and Tinea manuum in a descending order of frequency. In India the commonest species isolated were T. rubrum followed by T. mentagrophytes. E. floccosum is commonest species causing Tinea capitis. ${ }^{6}$

Primarily it depends on the habits and living condition of the people as it is transmitted through fomites. Sex, race and occupation have a little differential influence upon the frequency of Dermatophytosis. ${ }^{7}$

The risk factors include overcrowding, poor personal hygiene, immunosuppressive therapy, cancer chemotherapy and immunocompromised conditions. ${ }^{8-9}$

\section{Material and methods:-}

This prospective cross sectional study was conducted, after obtaining approval from institutional ethical committee and fully informed and voluntary consent were obtained from the patient and / or attendants. A total of 300 patients of suspected Dermatophytosis were enrolled as cases for the study which fulfill the inclusion criteria, attending skin OPD in J. A. Group of Hospitals of Gajra Raja Medical College, Gwalior, Madhya Pradesh were studied prospectively for study period of 1 year.Patient with co morbid condition and who was on antifungal drugs within 12 months and who was not willing to participate in the study are excluded.

Preferably two samples were collected from each patient for culture and $\mathrm{KOH}$ purpose. All the samples were taken and processed aseptically for direct $\mathrm{KOH}$ mount, PAS staining and culture on Sabouroud's dextrose agar with and without antibiotics and Dermatophyte test medium (DTM). ${ }^{10}$

One set was incubated at $37^{\circ} \mathrm{C}$ and other set at $25^{\circ} \mathrm{C}$ for 4 weeks and was examined daily for first week and then twice a week thereafter for any fungal

growth. Identification was done on the basis of colony characteristics as well as microscopic morphology in Lacto phenol cotton blue mount. One set of Dermatophyte Test Medium containing actidione to inhibit saprophytic fungi, gentamicin and chloramphenicol to inhibit bacteria was also inoculated and incubated at $25^{\circ} \mathrm{C}$ for 15 days for color change. The Dermatophytes turn the medium red at $25^{\circ} \mathrm{c}$, due to increased PH through their metabolic activity and indicated by the indicator phenol red present in the medium. While most other fungi and bacteria do not.

\section{Result \& Discussion:-}

Out of 300 Samples collected, Dermatophytes isolates were 159 (53\%).Non-dermatophytic moulds and candida species were $42(14 \%)$. This was similar to the study done by Ndako JA et al (2012) ${ }^{11}$ where they also found prevalence of dermatophyte in 53\% cases. In a study done by Vandana Upadyay et al in $2019^{12}$, prevalence of dermatophyte was 51\%. $210(70 \%)$ samples were positive by direct microscopy (KOH), 201 (67\%) samples showed growth in cultures. Among $210 \mathrm{KOH}$ positive samples 171 were also culture positive. In our study, Male: Female ratio was $2.75: 1$, showing male preponderance. Similar study done by Anusandanam Pavani et al (2016) ${ }^{13}$ from Mahabubnagar, found male:female ratio of 2.83:1. Most of the cases were seen between 21 - 30 years of age group. Ramana P.V et al (2017) ${ }^{14}$, Suruchi Bhagra et al (2014) ${ }^{15}$, Kamothi et al (2010) ${ }^{16}$ documented higher incidence of suspected dermatophyte infection in age group of 21-30 yrs.

Two genera of Dermatophytes were isolated. Trichophyton $(96.85 \%)$ species were the predominant isolates followed by Microsporum (3.15\%).

Trichophyton rubrum was the predominant species isolated from Tinea corporis, Tinea cruris, Tinea unguium and Tinea capitis. 
The other Dermatophytes isolated were Trichophyton mentagrophytes, Trichophyton verrucosum, Trichophyton schoenlenni, Microsporum gypseum and Microsporum canis.In vitro hair perforation test helps to differentiate Trichophyton mentagrophytes from Trichophyton rubrum and Microsporum canis from Microsporum equinum. Urease test help to differentiate Trichophyton mentagrophytes from Trichophyton rubrum.

\section{Graph 1 : Sex-wise distribution among the dermatophyte group}
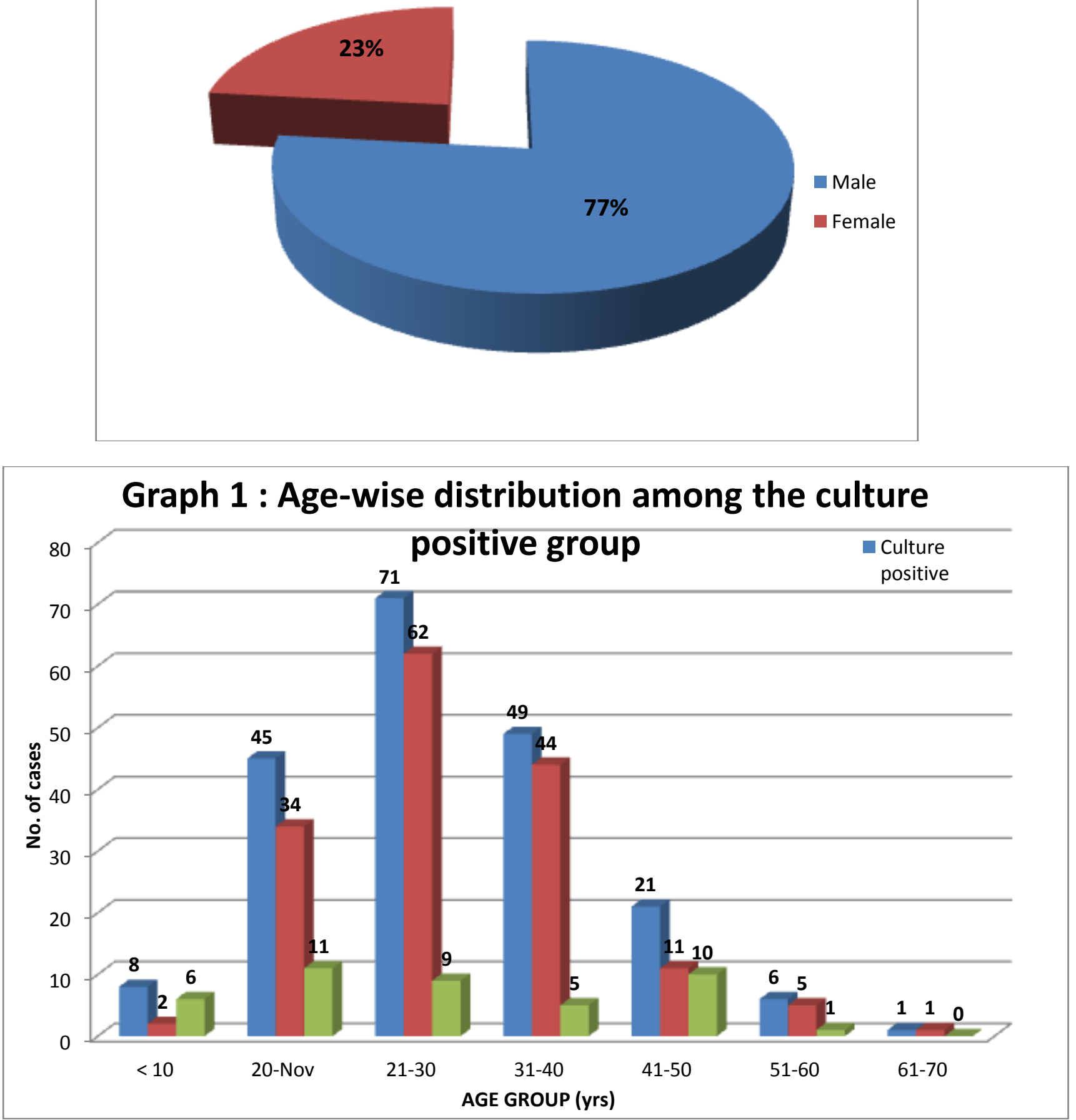
Table 1:- Distribution of cases according to clinical diagnosis.

\begin{tabular}{|l|l|l|l|}
\hline Samples & Dermatophyte & Number & Percentage \\
\hline \multirow{5}{*}{ Skin } & T.rubrum & 69 & $43.40 \%$ \\
\cline { 2 - 4 } & T.mentagrophyte & 41 & $25.79 \%$ \\
\cline { 2 - 4 } & T.verrucosum & 03 & $1.89 \%$ \\
\cline { 2 - 4 } & M.gypseum & 02 & $1.26 \%$ \\
\cline { 2 - 4 } & M.canis & 02 & $1.26 \%$ \\
\hline \multirow{5}{*}{ Nail } & T.rubrum & 19 & $11.95 \%$ \\
\cline { 2 - 4 } & T.mentagrophyte & 11 & $6.91 \%$ \\
\cline { 2 - 4 } & T.verucosum & 02 & $1.26 \%$ \\
\hline \multirow{5}{*}{ Hair } & T.rubrum & 05 & $3.14 \%$ \\
\cline { 2 - 4 } & T.mentagrophyte & 02 & $1.26 \%$ \\
\cline { 2 - 4 } & T. schoenlenni & 02 & $0.62 \%$ \\
\cline { 2 - 4 } & M.gypseum & 01 & 100 \\
\hline
\end{tabular}

\section{Conclusion:-}

Dermatophytosis are worldwide distributed with increased incidence especially in tropical countries like India.Several factors such as age, sex, poor hygiene influence the infection with Dermatophytes. Tinea corporis was the predominant clinical site from which Dermatophytes were isolated. T.rubrum and T.mentagrophytes were the major etiological agent. Preventive measures such as maintenance of personal hygiene, avoidance of tight and restrictive clothing and early diagnosis and treatment of clinically suspicious cases plays a major role in control of these infections.

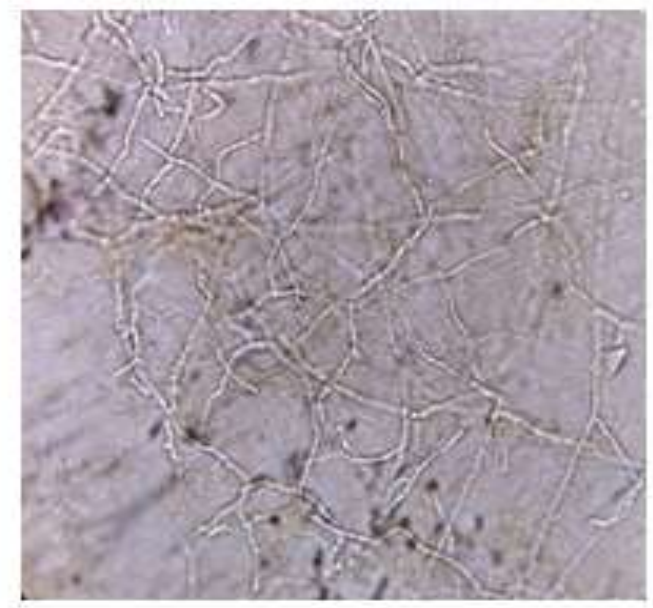

\section{Septate, hyaline hyphae on $\mathrm{KOH}$ wet mount (40 X)}



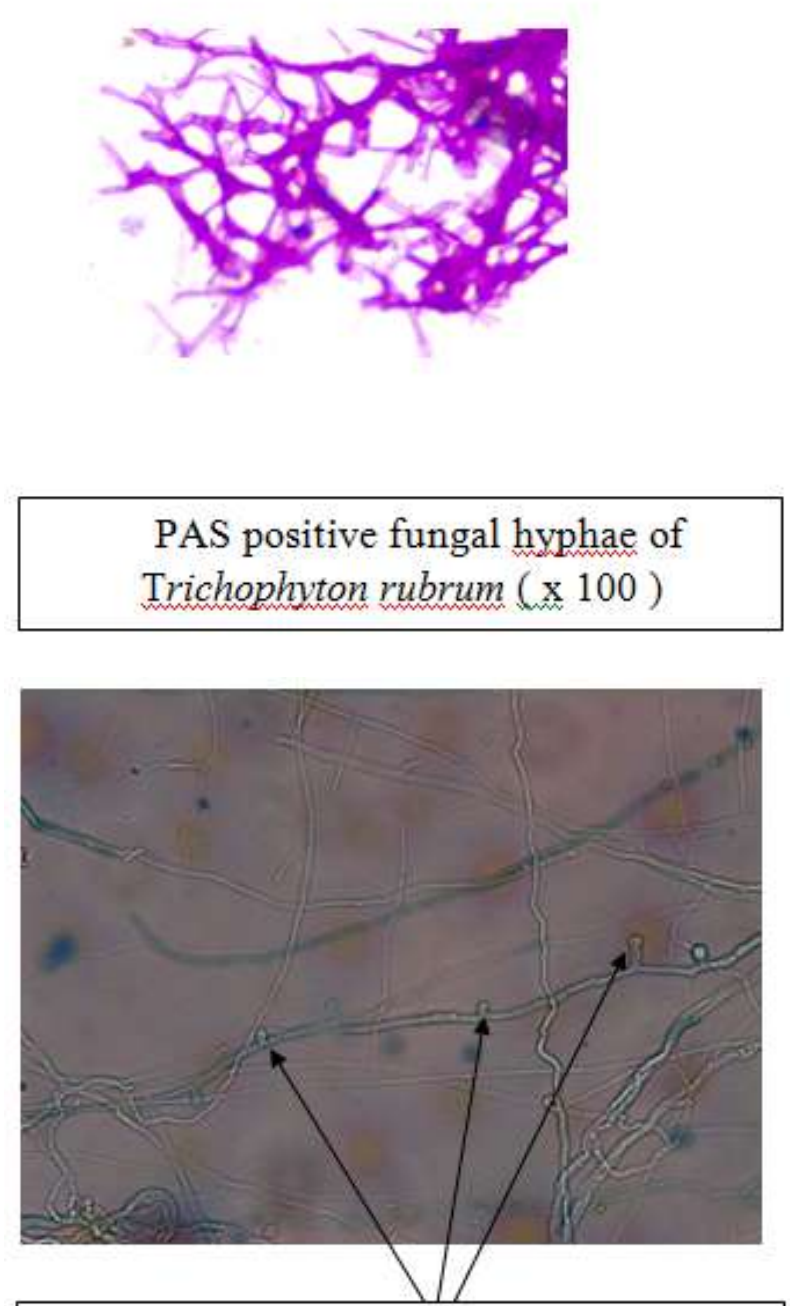

Bird $\mathrm{On}$ Fence appearance on LPCB mount of Trichophyton.rubram

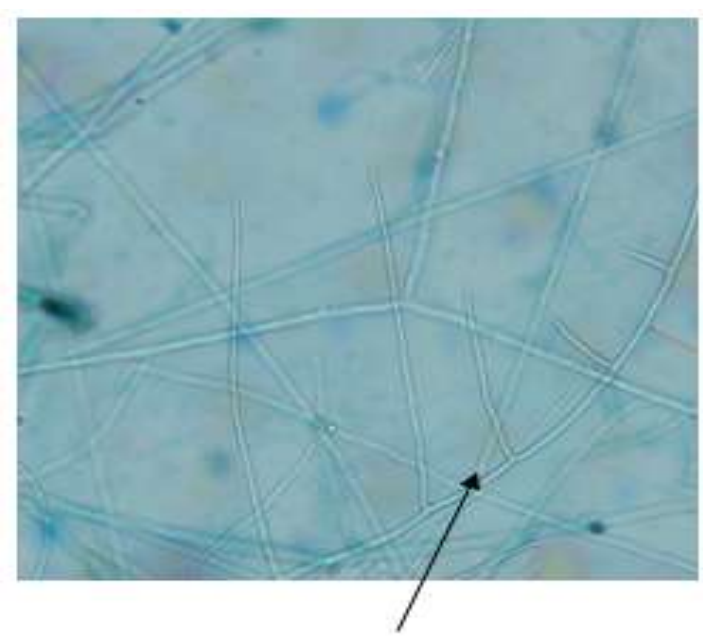

Pectinate Hyphae 


\section{Refeferences:-}

1. Jagdish Chandra, Textbook of Medical Mycology, $4^{\text {th }}$ Edition.

2. Tao Liu et al. Analysis of gene expression changes in Trichophyton rubrum after skin interaction. Journal of Medical Microbiology (2014); 63: 642-8.

3. Surendran KAK et al. A Clinical and Mycological Study of Dermatophytic Infections. Indian Journal of Dermatology 2014; 59(3):262-267.

4. Albert Balows BID. Topley \& Wilson's microbiology and microbial infections.Vol. 92, Transactions of the Royal Society of Tropical Medicine and Hygiene.1998.

5. Vijayakumar Ramaraj et al. Incidence and prevalence of dermatophytosisin and around Chennai, Tamilnadu, India. International Journal of Research in Medical Sciences 2016;4(3): 695-700.

6. Kanwar et al. IADVL Text book and Atlas of Dermatology, 2nd edition, Mumbai 2001, 21-58.

7. Ahmed Medhat Mohsen Hanafy et al Epidemiology of cutaneous mycosis in the Medina region of Saudi Arabia correlated with studying the effect of light-induced gold nanoparticles on the growth of Dermatophytes in vitro. African Journal of Microbiology Research 2012;6(37):6668-6677.

8. P Veer et al. Study of onychomycosis: prevailing fungi and pattern of infection. Indian Journal of Medical Microbiology 2007;25 (1):53-56.

9. R Kaur et al. Onychomycosis - epidemiology, diagnosis and management. Indian Journal of Medical Microbiology 2008;26(2): 108-116.

10. Gupta Sarika et al Prevalence of dermatophytic infection and determining sensitivity of diagnostic procedures. International Journal of Pharmacy and Pharmaceutical Sciences 2014; 6(3):35-38.

11. Ndako JA, Osemwegie OO, Spencer THI, Olopade BK, Yunusa GA, Banda J. Prevalence of Dermatophytes and other associated Fungi among school children. Global Advanced Res. J. Medicine and Medical Sci 2012; 1(3): 049-056.

12. Vandana Upadhyay et al, Epidemiological characterization of Dermatophytes at a tertiary care hospital in Eastern Uttar Pradesh, India, Current Medical Mycology 2019, 5(1): 1-6.

13. Anusandanam Pavani et al.Dermatophytosis in and around mahabubnagar. J. Evolution Med. Dent. Sci./ eISSN2278-4802, pISSN- 2278-4748/ Vol. 5/ Issue 32/ Apr. 21, 2016

14. Dr.P.Venkata Ramana et al. A Study of Dermatophytosis in Patients Attending Skin \& STD Outpatient Department at A Tertiary Care Government General Hospital And other Clinics in And Around Guntur, Volume 16, Issue 8 Ver. XI (Aug. 2017), PP 12-21.

15. Suruchi Bhagraet al, Mycological Pattern of Dermatophytosis in and Around Shimla Hills, Indian J Dermatol. 2014 May-Jun; 59(3): 268-270.

16. Kamothi et al.Prevalence of dermatophyte infection in district Rajkot, Electronic Journal of Pharmacology and Therapy Vol. 3, 1-3 (2010). 